\title{
A Call to Planetary2023 Panels to Implement Actionable Recommendations from Recent National IDEA Studies
}

This white paper is submitted as part of a collaborative effort organized by the Equity, Diversity, and Inclusion Working Group (EDIWG), a cross Assessment Group (AG) committee.

\section{Corresponding Author: Christina R. Richey, Jet Propulsion Laboratory, California Institute of Technology, (818)354-4940, christina.r.richey@jpl.nasa.gov}

Co-Authors:

Aparna Venkatesan, University of San Francisco, avenkatesan@usfca.edu

JA Grier, Planetary Science Institute, jgrier@psi.edu

Kartik Sheth, NASA Headquarters, kartik.sheth@nasa.gov

Maggie McAdam, NASA Ames Research Center, maggie.mcadam@nasa.gov

Mary Beth Wilhelm, NASA Ames Research Center, marybethwilhelm@nasa.gov

Matthew S. Tiscareno, SETI Institute, matt@seti.org

Meagan Thompson, NASA Headquarters, meagan.thompson@nasa.gov

Moses Milazzo, Other Orb, moses@otherorg.net

Parvathy Prem, Johns Hopkins Applied Physics Laboratory, parvathy.prem@jhuapl.edu

The Endorsement List contained 30+ names at the time of submission. The Endorsement form will stay open after submission.

Endorsements as of submission: Abigail Fraeman (JPL/Caltech), Veronica Allen (NASA GSFC/Catholic University), Mark A. Gurwell (Harvard \& Smithsonian CfA), Christian Schaller (LPL, UArizona), Matija Cuk (SETI), Vidhya Ganesh Rangarajan (University of Western Ontario), Kennda Lynch (LPI/USRA), Noam R. Izenberg (JHU APL, VEXAG Deputy Chair), Krista Soderlund (UT Austin), Barbara A. Cohen (NASA GSFC), Nicole Whelley (NASA GSFC/UMCP), Jennifer Scully (JPL/Caltech), Jacob Richardson (UMCP), Julie Rathbun (PSI), Nancy Chabot (JHU APL), Romy D. Hanna (UT Austin), Jennifer Hanley (Lowell Observatory), Linda Spilker (JPL/Caltech), M. Elise Rumpf (USGS Astrogeology Science Center), Jamie L. Molaro (PSI), Jeff Moore (NASA ARC), Alfred S. McEwen (UArizona), Terry A Hurford (NASA GSFC), Kunio M. Sayanagi (Hampton University), Evan L. Sneed (PSU), Margaret E. Landis (UCB/LASP), Carol Paty (UOregon), Gwen Hanley (US Berkeley), Edgard G. Rivera-Valentín (LPI/USRA), Morgan L. Cable (JPL/Caltech), Catherine Elder (JPL/Caltech), Zahra Khan (independent)

Acknowledgements: A portion of the work described was carried out at the Jet Propulsion Laboratory, California Institute of Technology, under a contract with the National Aeronautics and Space Administration (80NM0018D0004). 
Introduction: Task \#9 of the ongoing Planetary Science and Astrobiology Decadal Survey 2023 (hereafter Planetary2023) is to provide a clear exposition of "[t]he state of the profession including issues of diversity, inclusion, equity, and accessibility, the creation of safe workspaces, and recommended policies and practices to improve the state of the profession."1

While demographic representation in the community is the focus of several other white papers submitted to Planetary2023 (including Rivera-Valentin et al., Who is missing in Planetary Science: A Demographics study of the planetary science workforce ${ }^{2}$ and Rathbun et al., Who is Missing in Planetary Science?: Recommendations to increase the number of Black and Latinx scientists $^{3}$ ), understanding the workplace environments beyond diversity is needed to assess both inclusion and accessibility (c.f., Watkins et al., Professional development in the next decade: Supporting opportunities in all career paths and life events ${ }^{4}$ ). Inclusion, Diversity, Equity, and Accessibility (IDEA, also referred to as DEIA) as they apply to scientific disciplines such as planetary science, have been intensely studied by professional social scientists for decades. Major reports have been conducted by professional societies and the National Academy on topics that are highly applicable to Task \#9 of Planetary2023. Here, we summarize five major reports and studies since 2016 that address issues faced by those who self-identify as women, an underrepresented minority and/or LGBT. Our colleagues and students who have intersectional or additional marginalized identities bear the greatest fallout and are most at risk. We highlight the findings from these landmark reports that would be critical to push forward in the planetary science community. Note that each of these reports are hundreds of pages long and cannot be fully summarized in a seven page White Paper. However, we will highlight the major results and the recommendations from the reports to provide key information to the Decadal Committee.

Recommendation \#1: The Planetary2023 Decadal panel should carefully consider the findings and recommendations of these five critical reports as they apply to the fields of planetary science and astrobiology.

Recommendation \#2: As stated also in other White Papers being submitted, the Planetary 2023 panel should include the expertise of social scientists and others who have a track record of working to promote IDEA within the Planetary Science and Astrobiology community and/or within the STEM community more generally (see Bennett et al., The Preventing Harassment in Science Workshop: Summary and Best Practices for Planetary Science and Astrobiology ${ }^{5}$, or see Richey et al., Lessons Learned from the Astro Decadal Survey White Papers ${ }^{6}$, for more information). In order to help the panel with this, we will include as a separate google document link the full committee and author list of each national report in this White Paper?. These memberships can also be clearly found in the reports themselves. Note that committee

\footnotetext{
${ }^{1}$ https://www.nationalacademies.org/our-work/planetary-science-and-astrobiology-decadal-survey-2023-2032

2 Draft: https://drive.google.com/file/d/1n4UUIDV426ZwwTQ-0RfI5BtPOF40H5XM/view

${ }^{3}$ Draft: https://docs.google.com/document/d/1fQUB-PK47ecCvtnGeFAdSQIVILMyrqbQhUJw7oDj6uM/edit\#

${ }^{4}$ Draft: https://docs.google.com/document/d/1e_cOaCNSFlYYOh4DNo93aAYLDTC-_NicTVFifyx3Rgk/edit

${ }^{5}$ Draft: https://docs.google.com/document/d/1e gbHPUCANDOo6-PiZLdzNNt7YnwzHSwaTVbhdJoXTE/edit?ts=5f3c3fe3

${ }^{6}$ Draft: https://docs.google.com/document/d/12q413JMmMvFt4cgQflzglIPtVsvXvPwhiGRyXsvlcug/edit

${ }^{7}$ https://docs.google.com/document/d/11pcuha4N70MC47puc6kfAJEMG QvmnLKtWI8CVklitg/edit?usp=sharing
} 
and author membership were taken from the reports and affiliations and titles may have changed since the date of the report's publication.

Recommendation \#3: Important studies are only valuable if organizations are aware of them and then commit to implementing the recommendations contained in those studies. The Planetary2023 Panel should exhort NASA, affiliated partners, and contractors to develop and maintain the infrastructure necessary to ensure changes relevant to that specific Center or partner are made. Such a structure would include funding and empowering personnel to: remain current on relevant studies from within space science as well as the social sciences, draft recommendations from those studies applicable to the organization, work with all stakeholders to propose changes to the organization's policies and work environment, exercise oversight as necessary to ensure changes are implemented, and conduct evaluation through all stages to confirm the magnitude and nature of resultant change.

Report \#1: National Academies of Sciences, Engineering, and Medicine. 2018. Sexual Harassment of Women: Climate, Culture, and Consequences in Academic Sciences, Engineering, and Medicine. Washington, DC: The National Academies Press. doi: $10.17226 / 24994 .^{8}$

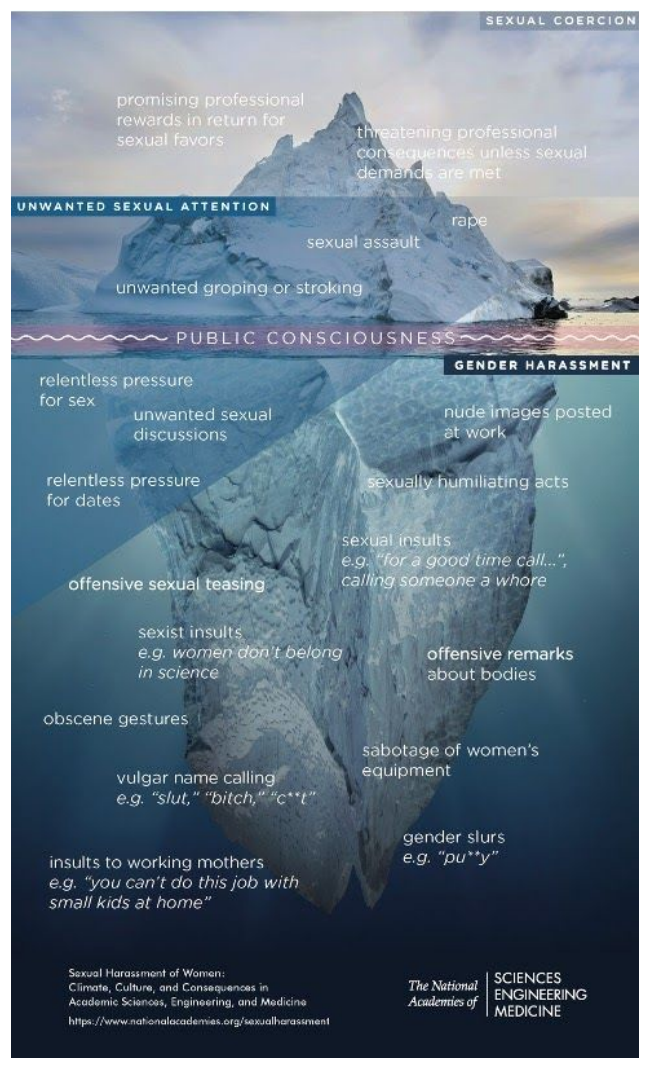

The National Academies of Sciences, Engineering, and Medicine Report on Sexual Harassment of Women: Climate Culture and Consequences in Academic Science, Engineering, and Medicine (2018) is the report of a special ad hoc study committee of researchers, academic and business leaders, and others with expertise on this topic to investigate the issue and how sexual harassment could be addressed. The Statement of Task was to undertake a study of the influence of sexual harassment in academia on the career advancement of women in the scientific, technical, and medical workforce.

\section{Major Findings from the Report:}

- $50 \%$ of women faculty and staff in academia experience sexual harassment.

-Between $20-50 \%$ of students in science, engineering, and medicine experience sexual harassment from faculty or staff.

-The cumulative effect of sexual harassment is significant damage to research integrity and a costly loss of talent in academic sciences, engineering, and medicine.

-The two characteristics most associated with higher rates of sexual harassment are (a) male-dominated gender ratios and leadership and (b) an organizational climate that communicates tolerance of sexual harassment

\footnotetext{
${ }^{8}$ https://www.nationalacademies.org/our-work/sexual-harassment-in-academia
} 
- Organizational climate is, by far, the greatest predictor of the occurrence of sexual harassment, and ameliorating it can prevent people from sexually harassing others.

\section{Recommendations from the Report:}

- Leaders in academic institutions and research and training sites must pay increased attention to and enact policies that cover gender harassment as a means of addressing the most common form of sexual harassment and of preventing other types of sexually harassing behavior. - Move beyond legal compliance to address culture and climate. Academic institutions, research and training sites, and federal agencies should move beyond interventions or policies that represent basic legal compliance and that rely solely on formal reports made by targets.

- Professional societies should accelerate their efforts to be viewed as organizations that are helping to create culture changes that reduce or prevent the occurrence of sexual harassment.

\section{Recommendations for Institutions from the Report:}

- Create diverse, inclusive, and respectful environments.

- Diffuse the hierarchical and dependent relationship between trainees and faculty.

- Provide support for targets.

- Improve transparency and accountability.

- Strive for strong and diverse leadership.

- Make the entire academic community responsible for reducing and preventing sexual harassment.

Report \#2: LGBT Climate in Physics: Building an Inclusive Community (American Physical Society, College Park, MD, 2016). ${ }^{9}$

An Ad-Hoc Committee reviewed the status of LGBT+ physicists to assess the barriers to full inclusion within the physics community. Input was obtained through focus groups held at APS meetings, a detailed climate survey, and a set of in-depth interviews with individuals who self-identify as LGBT.

\section{Major Findings:}

-The overall climate experienced by LGBT physicists was highly variable.

- In many physics environments, social norms established expectations of closeted behavior.

- LGBT physicists with additional marginalized identities faced greater level of discrimination.

- Transgender and gender-nonconforming physicists encountered the most hostile environments.

- Many LGBT physicists were at risk for leaving their workplace or school.

- LGBT physicists reported trouble identifying allies to help mitigate isolation, exclusion, or marginalization.

\section{Recommendations:}

- Ensure a safe and welcoming environment at meetings.

- Establish written best practices

- Implement Codes of Conduct

-Address the need to systematically accommodate name changes in publication records.

${ }^{9}$ https://www.aps.org/programs/lgbt/upload/LGBTClimateinPhysicsReport.pdf 
-Develop advocacy efforts that support LGBT equity and inclusion

-Promote LGBT-inclusive practices in academia, national labs, and industry.

- Develop training program on inclusive workplace and mentorship practices

- Utilize Climate Site Visit Programs.

-Implement LGBT-inclusive mentoring programs.

- Includes creating a professional network of LGBT mentors and mentees and hosting networking events.

- Support the establishment of a Forum on Diversity and Inclusion.

-Disseminate Best Practice Guide developed by LGBT+ physicists*

* One such document created based off of this recommendation is the LGBT+ Inclusivity in Physics and Astronomy: A Best Practices Guide (2nd Ed, 2018). ${ }^{10}$

Recommendations:

- Assess and address: participate in or conduct a climate survey, collecting demographic information, carrying out classroom climate assessments. Establish a departmental climate committee and/or liaison and explicit LGBT+ supportive policies.

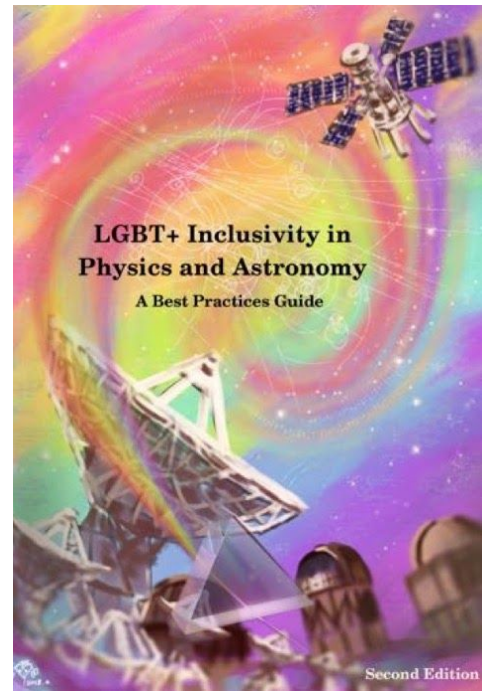
- Break the silence and invisibility: Initiate department-wide discussions of LGBT+ concerns, highlight the scientific contributions of LGBT+ department members at all levels, join an "Out List" as an ally or LGBT+ scientist, identify LGBT+ supportive mentors, invite LGBT+ speakers to campus.

- Educate and advocate: Participate in LGBT+ friendly climate and anti-bias training, work for campus-wide LGBT+ supportive practices such as supportive first responders and gender-inclusive restrooms and accompanying signage.

- Set the example and expectations: include preferred pronouns in your email signature, invite students and/or meeting participants to share their preferred pronouns, articulate classroom environment expectations on the first day of class, speak up in response to discriminatory behavior and report where appropriate.

- Support and include: Plan gender-neutral and inclusive social events, create LGBT+ safe spaces in your department, provide equal restroom access, include LGBT+ faculty in positions of authority, provide support for participation in LGBT+ networking events, ensure LGBT+ needs are considered in dual-career hires, family-friendly policies, and benefits.

Report \#3: The AIP National Task Force to Elevate African American Representation in Undergraduate Physics \& Astronomy (TEAM-UP). The Time is NOW: Systematic Changes to Increase African Americans with Bachelor's Degrees in Physics and Astronomy (American Institute of Physics, College Park, MD, 2020). ${ }^{11}$

The goal of this report is to motivate the key stakeholders to increase the number of African American students earning bachelor's degrees in physics and astronomy.

${ }^{10}$ https://aas.org/sites/default/files/2019-09/LGBTInclusivityPhysicsAstronomy-BestPracticesGuide2ndEdn small.pdf

${ }^{11}$ https://www.aip.org/sites/default/files/aipcorp/files/teamup-full-report.pdf 


\section{Findings:}

-The persistent underrepresentation of African Americans in physics and astronomy is due to:

-The lack of a supportive environment for these students in many departments.

-The enormous financial challenges facing them and the programs that have consistently demonstrated the best practices in supporting their success.

- Solving these problems requires addressing systematic and cultural issues, and creating a large-scale change management framework.

\section{Recommendations:}

- Fostering a sense of belonging is essential for African American student persistence and success.

- To persist, African American students must perceive themselves, and be perceived by others, as future physicists and astronomers.

- Faculty encouragement, recognition, and representation were key enablers.

- Effective teaching and strengths-based approach to academic support are necessary.

- Many African American students need support to offset financial burdens and stress.

- For sustainability, academic \& disciplinary leaders must prioritize creating environment, policies, and structures that maximize African American student success.

Report \#4: Promising Practices for Addressing the Underrepresentation of Women in Science, Engineering, and Medicine (STEMM): Opening Doors ${ }^{12}$ (NASEM, 2020)

This report draws on the work of two National Academies Committees: the Committee on Increasing the Number of Women in STEMM and the Committee on Understanding and Addressing the Underrepresentation of Women in Particular Science and Engineering Disciplines. The report emphasizes the experiences of women of color, and women with intersecting identities, and discusses differences in experiences between scientific disciplines.

\section{Findings:}

1. Women, especially women of color, are underrepresented relative to their presence in the US workforce and population. Representation varies by career stage, race and ethnicity, and discipline.

2. This underrepresentation is driven by bias, discrimation and harassment, which are more overt and intense for women with intersecting identities, such as women of color and women with disabilities or LGBTQIA identities.

3. Some institutions have seen improvements in representation over time, but underrepresentation of women remains nationally prevalent.

4. While there are numerous evidence-based strategies to improve recruitment, retention and advancement of White women, more work is needed to understand how to support women with intersecting identities more effectively.

5. Improving recruitment and retention throughout education and training is important, particularly in math-intensive fields. Challenging stereotypes about STEMM work and

12

https://www.nap.edu/catalog/25585/promising-practices-for-addressing-the-underrepresentation-of-women-in-s cience-engineering-and-medicine 
workers can increase interest, performance and sense of belonging among women and other underrepresented groups (such as first-generation students and men of color).

6. Institutional change requires committed leadership at all levels, dedicated financial and human resources, data collection, accountability, and an explicitly intersectional approach to addressing multiple, cumulative forms of bias and discrimination.

\section{Recommendations:}

1. Institutions must be transparent and accountable. In particular, the report outlines implementation actions for legislative and executive branches of government, and federal agencies in ensuring transparency and accountability in data collection and reporting, and analysis of progress.

2. Adopt data-driven approaches to address underrepresentation. The report recommends disaggregating data to analyze barriers by discipline, career stage, and intersecting identities (while protecting anonymity). Institutions should collect data, dedicate resources for qualitative research into institutional climate, and implement actionable, evidence-based practices in the areas of education, recruitment, and retention. The report emphasizes the importance of mentorship initiatives, direct and visible support for targets of sexual harassment, and counterspaces (physical or conceptual) that provide a sense of belonging. Federal agencies should support such initiatives and related research, and address funding disparities for women, especially women of color.

3. Reward, recognize and resource DEI efforts. Effective practices should be institutionalized, DEI initiatives should receive resources and authority at the institutional level and formal recognition (including consideration in professional advancement) at the individual level. Professional societies should do likewise. Federal agencies and private foundations should recognize and celebrate colleges and universities working to improve gender equity.

4. Fill knowledge gaps. Critical knowledge gaps exist with respect to intersectional experiences, strategies to support women with intersectional identities, factors contributing to the disproportionate benefit to White women from interventions, factors contributing to the success and failure of interventions at various institutions, long term evaluation of practices, best practices at non-research intensive institutions (such as community colleges), and characteristics of effective male allies and approaches to training.

Report \#5: Minority Serving Institutions: America's Underutilized Resource for Strengthening the STEM Workforce ${ }^{13}$ (NASEM, 2019)

This report makes the case that increasing the representation of students of color in STEM fields is critical to US national economic interests, and that Minority Serving Institutions (MSIs) have the ability to address current under-representation. The report focuses on the successes and challenges that MSIs have faced in preparing students to enter the STEM workforce, and identify actions that can be taken by the public and private sector, as well as MSI leadership.

\section{Findings:}

13

https://www.nap.edu/catalog/25257/minority-serving-institutions-americas-underutilized-resource-for-strengthe ning-the-stem 
Through a review of existing and commissioned research, and site visits, the committee found that a common thread running through the successes of MSIs was the intentionality with which they serve and support the success of their students. The report identifies the following promising strategies:

1. Dynamic, multilevel, mission-driven leadership, with vision and accountability.

2. Institutional responsiveness to meet students where they are, by providing academic, financial and social support and flexibility.

3. Supportive campus environments, with a sense of community and an equity-oriented culture.

4. Tailored academic and social support, including summer bridge programs and supplemental instruction.

5. Mentorship and sponsorship.

6. Availability of undergraduate research experiences, which have become increasingly important in STEM careers, sometimes offered in a course-based format or through external partnerships.

7. Mutually beneficial public and private sector partnerships with local and federal government, business, and other educational institutions.

\section{Recommendations:}

1. The leadership of MSIs should support a culture of intentionality, creating and sustaining evidence-based, outcome-driven programs to support student success.

2. MSIs should invest in succession planning and professional development for current and future leaders.

3. MSIs should establish and expand mutually beneficial and sustainable partnerships with the public and private sectors.

4. Public and private funding agencies should continue and expand grant programs that enable MSIs to recruit and retain faculty, maintain state-of-the-art labs and facilities, and offer extraordinary academic and social support services.

5. Recognizing the institutional resources that are required to effectively compete for large grants, funding agencies should consider the practicality of competitive funding models for under-resourced MSIs.

6. At the same time, MSI leadership should proactively position themselves to compete for grants, independently or through collaborations.

7. Funding agencies should expand and support evidence-based research into MSIs, their students and programmatic interventions.

8. Congress should enhance clarity, transparency and accountability in reporting on investments in STEM education and research at MSIs.

9. Congress should incentivize investment in MSIs and in the promising strategies identified above.

10. Measures of institutional success should be refined to account for institutional missions, investment in faculty, student populations and needs, and resource constraints. 